\title{
Cartografía y patrimonio en perspectiva
}

Gonzalo Acosta Bono, Instituto de Cartografía de Andalucía

Entre el documento histórico y la gestión del patrimonio, título de este monográfico dedicado a la Cartografía, resume el hilo argumental que recorre las diferentes secciones y colaboraciones. No es la primera vez que $\mathrm{PH}$ aborda cuestiones relacionadas con la cartografía y la información geográfica, y precisamente por ello se han ido creando las condiciones que hacen posible este número especial para aproximarnos a cada uno de los aspectos que interesan desde la politica del patrimonio cultural.

La relación entre Cartografía y cultura queda establecida desde su propio origen, siendo como es una forma de expresión tan antigua como el propio lenguaje, y común a todo tipo de sociedades incluso en las que no conocen la escritura. La historia de la Cartografía es expresiva no sólo de una actividad cultural en parte científica y a la vez artística, sino también de la percepción subjetiva del mundo que corresponde a los contextos sociales de un periodo y un lugar concretos. La Cartografía ha desarrollado un lenguaje propio, un código de información gráfica (signos, símbolos y grafismos) para resolver las limitaciones impuestas por la necesaria conversión de la visión panorámica y volumétrica a un plano de dos dimensiones y por escala.

Mientras que la primera cuestión es un problema geométrico al que se han dedicado preferentemente cartógrafos y geodestas, la segunda conduce a una selección de datos que refleja una determinada percepción de la realidad. Estas limitaciones empiezan a superarse con las técnicas de la información geográfica y su enorme capacidad de acumular datos diversos, volviéndose a presentar la naturaleza común de ambos factores: Ios datos (información temática abierta a todas las disciplinas y usuarios) espaciales (un valor posicional preciso con arreglo a un sistema de referencia común). En cualquier caso, el quehacer cartográfico consiste en simplificar, reducir, estabilizar la realidad, y por tanto nada ajeno a los filtros de la interpretación de la realidad: en el ser humano se producen mecanismos que preceden a la capacidad de desarrollar y aplicar dicho código gráfico creado para expresar informaciones espaciales.

\section{EN EL PRINCIPIO ERA EL MAPA MENTAL}

Desde los primeros procesos de civilización humana ha existido la necesidad de construir un marco de referencias en el espacio. Bajo diferentes formas de expresión gestual y oral, y de simbolización de la realidad, se han desarrollado nociones espaciales como las de extensión, proximidad, dirección, distancia, sucesión, continuidad o itinerarios, tan necesarios para comunicar fuentes de recursos alimenticios, lugares de abrigo o para avisar de peligros. Con estas nociones basadas en la experiencia perceptiva se construyen los "mapas mentales" que el ser humano aprende para poder desenvolverse en su hábitat; es decir, ordena y sistematiza dichas experiencias que traduce en conocimiento. La sociedad misma está fundada sobre un espacio que aprende a controlar desde la comprensión de sus elementos y su organización, estableciendo límites y memorizando todas las referencias que aportan seguridad para su desarrollo. En todas las comunidades su lenguaje, en el sentido más amplio, revela la percepción ${ }^{1}$ que tiene de su espacio vital conformando su imaginario colectivo, como reflejan sus tradiciones y convenciones orales. Es decir, precede a la propia escritura cuyo proceso de fijación ha requerido, sin duda, elaboraciones más complejas. De hecho, ciertas escrituras conservan en su expresión ideográmica la visión plana o en perspectiva de los elementos del entorno ambiental que representan (FREITAG, 1980: 63). Nuestras propias representaciones del espacio nos remiten a la memoria de las primeras percepciones, que tienen en el cuerpo su referencia y alrededor del cual se organiza el mundo. La anatomía humana, con sus formas, simetrias y bilateralidad, nos presta las nociones que configuran el espacio como construcción mental ordenada: dentro/fuera, delante/detrás, izquierda/derecha, vertical/horizontal, etc. y todas las que de ellas se derivan².

Desde el cuerpo se adquiere conciencia de la posición central desde la que se organiza su rededor, el espacio exterior, cualquiera que sean sus dimensiones. En su oposición al espacio que se extiende en todo su alrededor, aqui y allá, se va estableciendo una relación dinámica, con el cerca y el lejos, mediante recorridos de ida y vuelta al centro. Orientación y dirección serán necesarias para cualquier clase de desplazamiento que va en- 
sanchando los límites conocidos. Cuando la experiencia adquiere ciertas dimensiones se construyen referencias cada vez más generales y abstractas, aunque la más elemental, la de los cuatro puntos cardinales en que se divide la circunferencia del horizonte, remite a los dos ejes que se proyectan desde nuestra centralidad corporal. La noción de "orientación", para aplicarlo al proceso intelectual, está hábilmente desarrollada en Kant, si bien su descripción es netamente geográfica por lo que resulta pertinente aquí. Está basada en la necesidad de "experimentar el sentimiento de una diferencia dentro de mí mismo: la diferencia entre la mano derecha y la mano izquierda, [para concluir que]... a pesar de todos los datos objetivos en el cielo, sólo me oriento 'geográficamente' gracias a un principio de diferenciación 'subjetivo' (ALMEIDA, 1998: 10).

Estos mapas mentales que manejan nociones espaciales, expresadas igualmente en el lenguaje, preceden al ejercicio cartográfico, entendido como la habilidad para producir, mediante el uso de re-

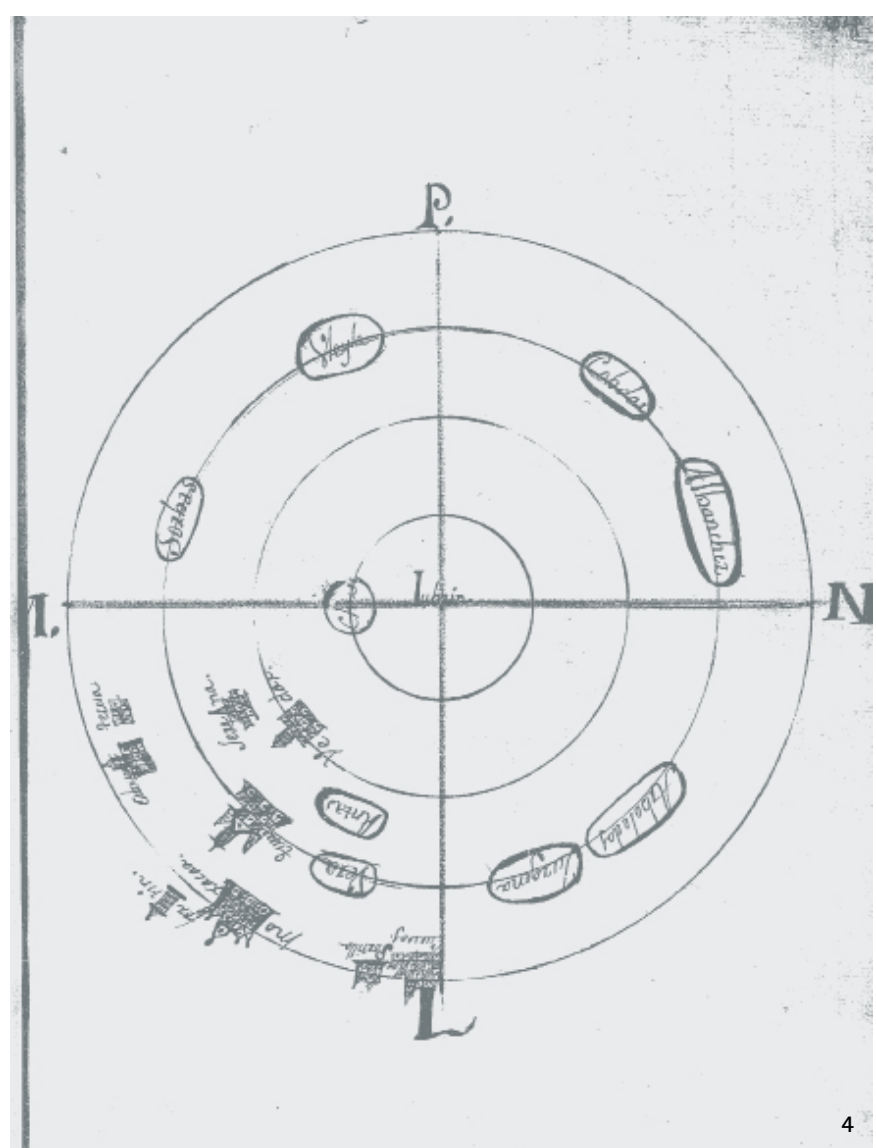

Distintas concepciones del espacio y su representación gráfica en respuesta a la encuesta de Tomás López para su Diccionario Geográfico: 1. El entorno de Pruna (Sevilla) se refleja en el propio ojo que lo mira. Fuente: Pruna (Sevilla). Dn. Juan Agustín Romero, cura. 1786. Biblioteca Nacional-Mss/7306-fol. 325, r.; 2. Un diagrama 3D, minucioso y preciso, para los alrededores de Bornos (Cádiz). Fuente: Bornos (Cádiz). Franco. Suarez de Vega. 1787. Biblioteca Nacional-Mss/7294-fol. 138, r.: 3. La escritura que describe Casarabonela (Málaga), con su disposición y caligrafía, dibuja su morfología configurando una imagen al estilo de la poesía visual. Fuente: Casarabonela (Municipio, Málaga). Dn. Antonio García Donoso natural y cura de la Yglesia Parrogl. de Santiago. 1780. Biblioteca Nacional (Madrid) -Mss/7303-fol. 71،

.; 4. Desde la centralidad de Lubrín (Almería), las referencias de su entorno no son topográficas (caminos, cursos de agua...), más bien topológicas. Fuente: Lubrín (Almería). Juan Ignacio Gallardo. 1774. Biblioteca Nacional (Madrid) -MSS/7294-fol. 64,5 

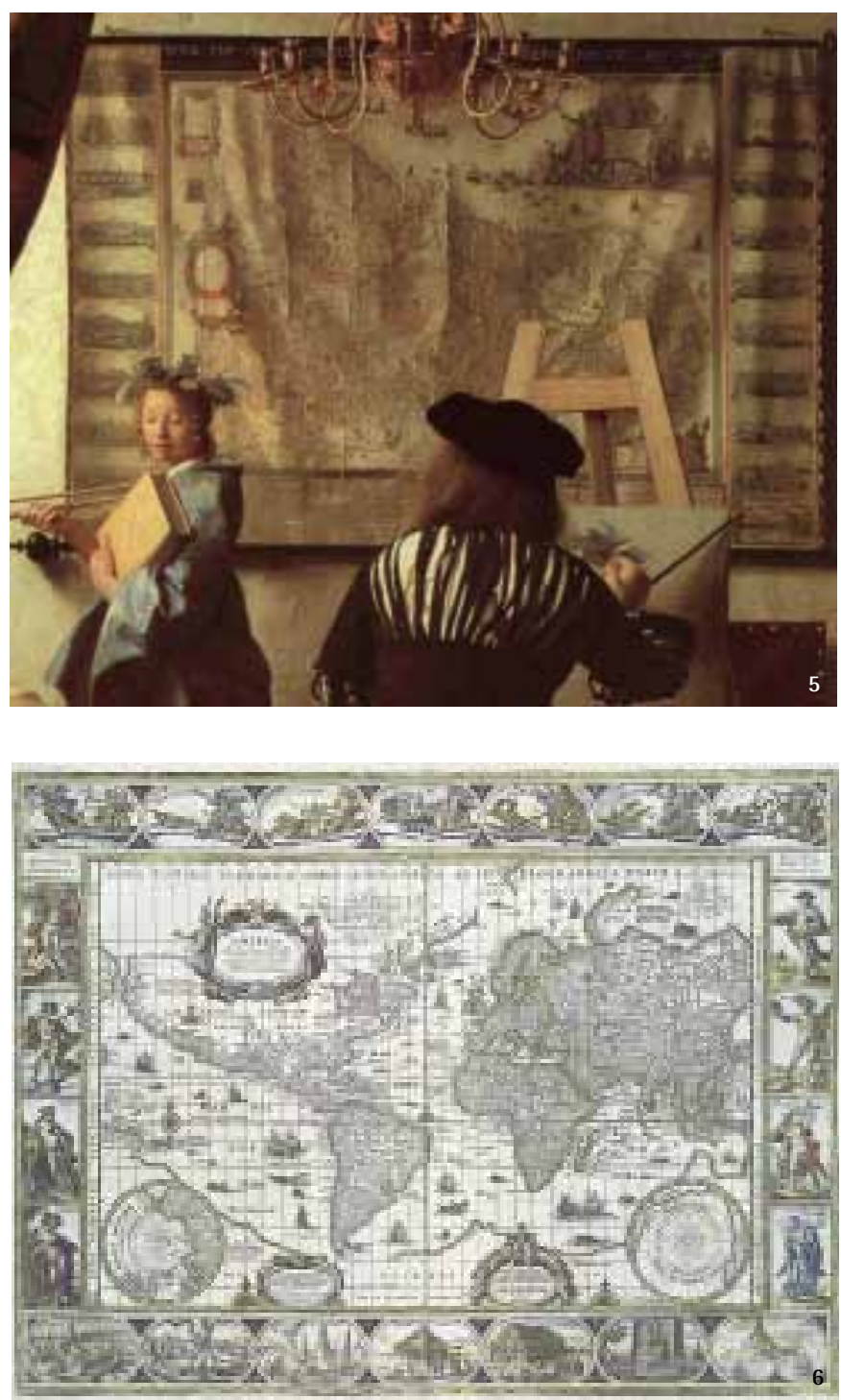

5. El mapa de este cuadro, El arte de la pintura, es obra de Claus Jansz Visscher (Piscator) y representa a los Países Bajos con vistas urbanas en los laterales de las 17 provincias. En la datación del cuadro (1673, en los inicios de la guerra franco-holandesa) ha sido clave la información que aporta el mapa, así como otros elementos que connotan el momento político. Fuente: SCHNEIDER, N (1994) Vermeer. La obra completa, pintura. Colonia (Germany): Taschen, 1994 6. Atlas Maior. Johannes Blaeu, 1659. Fuente: Biblioteca de la Universidad de Sevilla. Fondo Antiguo A215/135-144

cursos gráficos, los mapas que representan la realidad física, pero también simbólica. Mapas mentales, lenguaje y representación ponen de relieve las diferencias culturales que existen entre las diferentes sociedades y grupos sociales para expresar los elementos y relaciones que se dan en el espacio en el que se desenvuelven sus vidas. Así, puede constatarse estas diferencias entre sociedades nómadas y sedentarias, cazadoras y agrícolas, marineras y montañesas, desérticas y boscosas, autosuficientes y excedentarias, rurales y urbanas. Cada una de ellas -y se podría desagregar y matizar hasta particularizar en grupos locales- pone de relieve las diferencias culturales en la percepción y representación del espacio. Estas capacidades, de hecho, están en la base de informaciones contenidas en los mapas elaborados con técnicas avanzadas, sin las cuales quedarian reducidos a sus contornos geométricos. No son pocos los casos documentados de mapas basados en estas informaciones recabadas de los grupos locales aunque después la perspectiva et- nocentrista los ha tergiversado y ha ocultado su origen. Nombrar un lugar es tomar posesión de él (ZUMTHOR, 1994: 54), fijarlo en el idioma mediante un topónimo; las percepciones y conocimientos de un lugar tienen una gran carga semántica, de ahí que la toponimia contribuya significativamente al sentimiento de pertenencia. Pero por la misma razón también opera en los procesos de apropiación territorial. Tal es, y no otro, el sentido de muchas de las expediciones que han ido "descubriendo nuevos mundos", como si antes no existieran alli seres humanos que lo habitaran ${ }^{3}$. Más aún, la propia selección del espacio a cartografiar (el mundo físico) ya comporta una representación mental, subjetiva, de tal modo que entre ésta (la imagen mental) y la representación cartográfica (la imagen sobre el papel) existe una correspondencia 1:1, esto es, ajustada al esquema previo. En la simplificación de la realidad, desde su interpretación y reducción a lo significativo, operan presunciones, conjeturas y señales que van configurando nuestro modelo. Es sugerente, no obstante, la idea borgiana de que el trabajo cartográfico llegue a tal exhaustividad que acabará por rellenar el modelo ocupando todo el territorio: un mapa fractal llevado a su extremo acabaría por ocupar todo el territorio ${ }^{4}$.

Un caso excepcional de diversidad de criterios de representación del espacio vivido se lo debemos a Tomás López (Madrid, 17301802) y a su Diccionario Geográfico de España, empresa de corte ilustrada que no llegó a concluir. Para ella puso en marcha la recogida de información mediante cuestionarios enviados a los párrocos de los pueblos, entendiendo que eran las personas más cualificadas para este menester. Junto a los datos descriptivos se solicitaba "formar una especie de mapas o planos de sus respectivos territorios, de dos o tres leguas en contorno de su pueblo... nos contentamos con solo una idea o borrón del terreno..." La respuesta a tan insólita solicitud era previsible: sólo la mitad de los encuestados, que no son pocos dada la dificultad, y una información heterogénea, imprecisa y de dificil integración cartográfica. Se explica, en cierto sentido, que los diccionarios geográfico-estadisticos posteriores renunciaran a la representación gráfica de lugares y villas. Más allá de los propios datos y descripciones, el medio millar de gráficos, dibujos, perspectivas, esquemas, etc. constituye una colección de enorme valor porque ilustran los procesos con los que construimos la realidad, la percepción del espacio vital mediante la selección de sus elementos más significativos (objetos, toponimia, recorridos, límites, etc.) y los recursos gráficos empleados. Todo está connotado y ofrece múltiples detalles para la interpretación que se hace de la realidad ${ }^{5}$. Los mapas, en general, se convierten en metáforas espaciales, pero más libres cuantos menos sujetos están a las convenciones ${ }^{6}$.

\section{ENTRE EL RIGOR Y LA FANTASÍA: CONTEXTOS}

La experiencia de trasladar a un mapa las nociones espaciales estuvo durante la Edad Media organizada por símbolos y valores, frecuentemente religiosos, y su resultado fue una cartografía teo- 
lógica, como los famosos y esquemáticos mapas-mundi "T en 0 " (OJEDA ZÚJAR, 2000: 24-25). Los avances que se produjeron en el mundo clásico a partir de los cálculos astronómicos para determinar la posición fueron retomados por la cartografía islámica potenciada con los estudios en diferentes ciencias, entre ellas la Geografia, y las técnicas de navegación. En los siglos XIV-XV se produjo en Europa un importante cambio en la concepción del espacio, dejando de ser concebido como una jerarquía de valores para ser sustituido por el espacio como sistema de magnitudes (MUMFORD, 1977: 35). Mumford identifica como uno de los motores de este cambio el descubrimiento de la perspectiva y su aplicación a la pintura, sistematizada a partir de ésta en diferentes planos que ofrecen la sensación de profundidad y distancia. Ya no se trata de una relación simbólica sino visual entre los objetos y su representación, es decir, una relación medida, proporcionada, a escala. La pintura empieza a interesarse por el exterior introduciéndose paulatinamente en la composición del cuadro. Las consecuencias de este hallazgo no se limitan a la pintura artística; va a permitir también dibujar la arquitectura, no sólo la existente, también la que se proyecta para ser construida, dando lugar a numerosos tratados profusamente ilustrados sobre las técnicas arquitectónicas. En definitiva, un contexto en el que convergen las ciencias y las artes plásticas, entre el rigor y la fantasía, en el que la Cartografía participa de ambas conjeturando sobre el mundo físico y su representación.

Esta estrecha relación explica que pintores y cartógrafos compartieran durante mucho tiempo el mismo gremio, el de san Lucas. Las técnicas de impresión de mapas, el buril o la xilografía, ejercieron influencia en la pintura, tanta como la pintura en los códigos gráficos y en la composición de mapas. Sirva para ilustrar este hecho la colaboración de Hieronymus Cock, artista y grabador flamenco, y el cartógrafo Abraham Ortelius, autor de Theatrum Orbis Terrarum (Amberes, 1570), el primer atlas en sentido moderno; y también con el cosmógrafo de la Casa de Contratación de Sevilla Diego Gutiérrez, autor del espectacular mapa de América de 1562 (Americae sive quartae orbis partis nova et exactissima descriptio). Un siglo más tarde esta convivencia bajo el gremio de san Lucas se simboliza en quien fuera dos veces su decano, Jan Vermeer (1632-1675), artista que incorpora en su obra motivos cartográficos. Por entonces, Amberes era el principal centro productor, editor y distribuidor de mapas, atlas y globos terráqueos, que llegaron a convertirse en objetos apreciados por los grupos dominantes (visibles en la rica ornamentación, dedicatorias, etc.), y asociados a la mirada hegemónica que se tiene de un mundo dominado por las potencias europeas.

La evolución de la Cartografía corre paralela, sin duda, a la historia del conocimiento y sus aplicaciones técnicas, cuyos avances más notables están ligados a viajes y exploraciones de nuevos territorios, tanto para facilitar sus desplazamientos como para registrar la información de los nuevos lugares. A impulsos de los intereses expansionistas de las principales potencias (económicos, comerciales o políticos, y con frecuencia entrelazados), geógrafos y cartógrafos acompañaban a las expediciones, casi siempre. No debemos olvidar que descripción (geográfica) y representación (cartográfica) estuvieron unidas hasta el siglo XIX en el que la especialización del conocimiento las conformó como disciplinas diferentes ${ }^{7}$. La historia de la Cartografia ha primado esta perspectiva de los avances científicos: la brújula marina en el siglo XIII, la exactitud posicional, la representación plana de la esfera terrestre, el impacto que supuso la imprenta para la reproducción de mapas y atlas como objeto de consumo...

Ahora bien, hay otra forma de entender la Cartografia, de la que Brian Harley es su principal exponente. A través de sus investigaciones nos ha legado una manera de entender la historia de la Cartografía en claves culturales, sociales y políticas, y de forma nada ingenua, como instrumento de poder utilizado para el control de territorios, recursos y personas. A la pretendida objetividad y neutralidad de la las técnicas -obsesión propia de la cultura occidental- no ha escapado la Cartografía, corriente contra la cual ha argumentado con solvencia para devolvernos una nueva naturaleza de los mapas 8 . Hasta tal punto, que se ha adoptado el título de esta obra suya para caracterizar un cierto estilo de investigación sobre la Cartografía en la que prevalece la idea de cómo comprender el significado de los mapas en un sistema abierto en el que influye decisivamente todo el entramado de relaciones económicas, sociales y técnicas. En esta forma de entender los mapas, y en definitiva la imagen del mundo, intervienen además ideas de la Historia del Arte, la iconografía, la Literatura, la Semiótica o la Filosofía, que se oponen a la supuesta asepsia científica. Dice Harley (2005: 80) rotundamente: "Tanto en la selectividad de sus contenidos, como en sus signos y estilos de representación, los mapas son una manera de concebir, articular y estructurar el mundo humano, que se inclina hacia, es promovido por, y ejerce una influencia sobre grupos particulares de relaciones sociales". Desde hace varias décadas se multiplican los trabajos desde esta nueva epistemología que reflexiona sobre la visión del mundo que encierran los mapas, y no sólo lo que los mapas dicen del mundo. Sus aportaciones están desarticulando ciertas ideas sobre la Cartografía como un hecho ajeno a las condiciones en las que se produjeron. No es de extrañar, por eso mismo, que la riqueza de estos estudios provenga del continente americano, tan profusamente cartografiado por ser espacio donde se han desplegado todas las estrategias e instrumentos de dominio colonial, y también la Cartografia?.

Reconocerse en el espacio que define el ámbito vital, y el de la comunidad, forma parte de los procesos de identidad. La comunidad unifica la diversidad en relación con los otros en diversos ámbitos (local, nacional, grupos étnicos...), aunque es vieja la polémica sobre si el límite comporta la diferencia, o si la diferencia suscita el límite. El hecho es que el territorio es el espacio estructurado a partir de las relaciones sociales que se establecen para satisfacer las necesidades de una comunidad. Ahora bien, estos territorios evolucionan en la historia mediante procesos, circunstancias y vicisitudes complejas, en la misma medida que lo hace la propia sociedad. El territorio es también una construcción ideo- 
lógica cargada de atributos simbólicos, más cuanto participan de una cierta idea inmanente o esencial para fundamentar las bases territoriales de una estructura política. Es el caso de los Estados centralizados europeos a cuyo servicio también pusieron la Cartografía definiendo fronteras y levantando acta de sus posesiones. La historia de la Cartografía informa de estos procesos y aporta conocimiento para el relato histórico en toda su dimensión; pero también puede ser utilizada como prueba para construir identidades a partir de una visión mistificada de un territorio en el que procesos encadenados tienen como destino histórico conformar un espacio político con fronteras inalterables ${ }^{10}$.

Este tipo de tergiversaciones ha provocado reacciones desde diferentes experiencias, a la vez sugerentes y consecuentes, que tienen en común cuestionar el "orden" establecido. Contra el mapa es el título de un ensayo (DE DIEG0, 2009) que, desde el campo del arte, pone en entredicho la neutralidad de los mapas y reivindica propuestas provocadoras como la del dibujo de Joaquín Torres Garcia, un artista uruguayo que en 1935 cuestionó el norte convencional invirtiendo la representación del continente suramericano. Un precedente de una propuesta cartográfica, también de latitudes "sureñas", como el planisferio del australiano Stuart McArtur, editado en 1979 y al que siguieron otros muchos "mapas al revés" (LÓPEZ-GALIACHO, 2010). Pero, sin duda, el mayor impacto le corresponde a los mapamundis que utilizan la proyección del alemán Arno Peter (1974), directamente opuesta a la de Mercator (1569), la más generalizada de todas, la que consideramos tan "normal" que cualquier otra nos desorienta, por ejemplo la que ofrece el logotipo de la ONU que sitúa el centro de la imagen en el polo norte. Ninguna proyección está exenta de errores en la difícil traslación de un mundo elipsoidal a un plano bidimensional lo que se cuestiona no es de naturaleza cartográfica, sino política y social. Mercator sitúa a Europa en el centro del mapamundi, en realidad del mundo, y en consecuencia la línea del Ecuador -contradiciendo a su propia etimología- no divide la tierra en dos mitades iguales. En el planisferio de Peter, por el contrario, continentes y paises guardan unas proporciones más equilibradas, a costa de la preponderancia de Europa, ahora visiblemente disminuida. Es esta la razón por la que la UNESCO y muchas ONG la han adoptado para su representación del mundo.

Todos los mapas y planos producidos a lo largo de la historia representan un legado de gran valor documental y un caudal de información. Se señala certeramente, no obstante, que esta renovación conceptual y metodológica se limita a la historia de la Cartografía y a los mapas históricos, pero que no se aplican a los procesos actuales de elaboración de mapas, en su contexto sociocultural y en el mundo de la comunicación y la imagen tan desarrollado. Ciertamente, se producen cambios en las formas y retóricas, pero son igualmente aplicables a la actual producción de información geográfica. De momento, estamos en una fase de descubrimiento, desbordados por todas las potencialidades que ofrecen los nuevos recursos tecnológicos aplicados a la Cartografía en un sentido contemporáneo, tales son las radicales transformaciones que en los últimos años estamos verificando. Una de las características de este reciente proceso es su total "democratización", es decir, lo opuesto a un modelo de producción centralizado, basado en el control directo de los recursos técnicos y circunscrito a un estrecho grupo de especialistas. La Geografía y la Cartografía están en la calle, y por consiguiente también en cualquier ámbito disciplinar y de gestión temática, entre ellos el del patrimonio cultural.

\section{DEL MAPA A LA INFORMACIÓN GEOGRÁFICA}

El recorrido histórico de la Cartografía va de la mano de la evolución de las técnicas aplicadas al amplio mundo de la producción de mapas y planos. A los avances que supuso la sustitución del ojo humano por la visión fotográfica aérea vertical, más tarde enriquecida con técnicas para la detección de elementos y factores no perceptibles a simple vista (falso color, imágenes multiespectrales desde satélites, etc.), hay que añadirles las innovaciones que han supuesto la Electrónica y la Informática. Pero éstas trascienden a los meros cambios tecnológicos, por complejos que sean: con ellas estamos asistiendo a una verdadera renovación de los conceptos tradicionales. El mapa sobre soporte papel para ser observado e interpretado contiene toda la información que es capaz de ser representada: es una imagen analógica y abstracta, limitada por su carácter "fatalmente" bidimensional y el factor escala, y, en si mismo, el fin último del proceso cartográfico. Ahora el mapa es sólo un recurso de expresión gráfica de una información espacial que está almacenado en soporte digital; y evolucionando hacia las bases de datos geográficas (BDG), capaz de explotar cualquier dato que contenga un registro de su localización en el espacio, y no necesariamente en forma de coordenadas $(x, y)$. Esta trayectoria está suponiendo, por una parte, elevar a $n$ potencia la información a manejar, y por otra, a ampliar notablemente la base de usuarios, lo cual significa capacidad para gestionarla y, en definitiva, de incorporar valor añadido.

No se entendería este nuevo paradigma sin la red Internet, uno de los pilares de la sociedad de la información y el conocimiento y de la proyección de su futuro. De la alianza entre el mundo SIG, el de la organización y gestión de la información, y el mundo Web, surgen las mayores potencialidades de algo que sólo eran tendencias hace muy poco tiempo, pero que hoy vamos constatando avances muy logrados en todos los ámbitos. De un lado, la creciente capacidad de computación y de conectividad, para la cual es necesario el desarrollo de estándares que permitan la interoperabilidad. De otro, una nueva cultura de entender el trabajo en red basado en procesos compartidos y cooperativos, siendo su expresión más significativa el debate sobre los desarrollos en software libre y sobre licencias de uso que propicien la reutilización de la información y añadir valor creativo. Nueva cultura que ya está suponiendo un crecimiento exponencial de la información y el conocimiento, cuya expresión más acabada es la Web 2.0. Estas transformaciones no sólo tienen que ver con las tecnologías de la información y la comunicación. También han adquirido 
naturaleza jurídica, se han convertido en derechos para el conjunto de la sociedad, como así se manifiesta en la legislación más reciente: derechos de acceso a la información, de participación pública y acceso a la justicia; de acceso electrónico a los servicios públicos; sobre reutilización de la información del sector público ${ }^{11}$.

Estas constantes y aceleradas innovaciones atañen a todas las fases del proceso cartográfico. En un principio afectaron de forma superficial, aunque contribuyeron significativamente a la mejora de algunos aspectos (automatización del dibujo, por ejemplo); pero han acabado por revolucionar cada uno de los aspectos concernidos en la gestión de la información geográfica, concepto éste que adquiere centralidad y se desarrolla en cada de una de las fases de un proceso mucho más complejo: fuentes $>$ datos $>$ organización $>$ edición $>$ difusión. La conclusión es definitiva: mapas y planos tal como los hemos entendido hasta hace bien poco quedan desplazados a una fase muy determinada, como una de las opciones de edición o representación de la información geográfica. Esta visión es la que ofrece, en línea con las directrices europeas, el Plan Cartográfico de Andalucia, instrumento para el desarrollo y aplicación de la política de información geográfica en nuestra Comunidad.

\section{NUEVAS FUENTES PARA LA CAPTURA DE DATOS GEOGRÁFICOS}

Los instrumentos de observación han estado intimamente ligados a la capacidad tecnológica de cada momento, siendo la fotogrametria y la teledetección hitos que han definido la Cartografía contemporánea tanto topográfica como temática. El paso de la fotointerpretación y restitución analógica a los sistemas digitales ha supuesto un incremento considerable en la resolución y calidad geométrica, así como en la diversificación de los productos derivados de su explotación: modelos digitales del terreno y ortofotomapas. Hay que señalar, asimismo, que la componente posicional ha ganado en rigor, pero también en flexibilidad y variedad en su gestión, por ejemplo, mediante las operaciones automáticas de transformación de coordenadas. La progresiva implantación del GPS -de origen militar pero hoy felizmente "civilizada"- es uno de los factores responsables de la democratización de la captura de coordenadas y su aplicación a los más variados ámbitos. Con esta tecnología, que requiere ciertas cautelas en su uso, se está levantando cartografía de precisión más que suficiente, por personas y entidades sociales muy diversas.

Por su parte, la teledetección, el reconocimiento del territorio por medio de sensores remotos, ha supuesto una radical innovación en la captura de información, especialmente útil para la recogida de datos temáticos y, por la gran capacidad de computación, para generar inventarios, elaborar modelos de análisis y predicción, o realizar un seguimiento en tiempo real de procesos dinámicos. También ha permitido que la realidad analizada no se limite al ámbito terrestre, sino que se ha internado en los medios atmosférico y submarino. De este último es un buen ejemplo la explotación de la información geográfica para la gestión de los recursos patri-

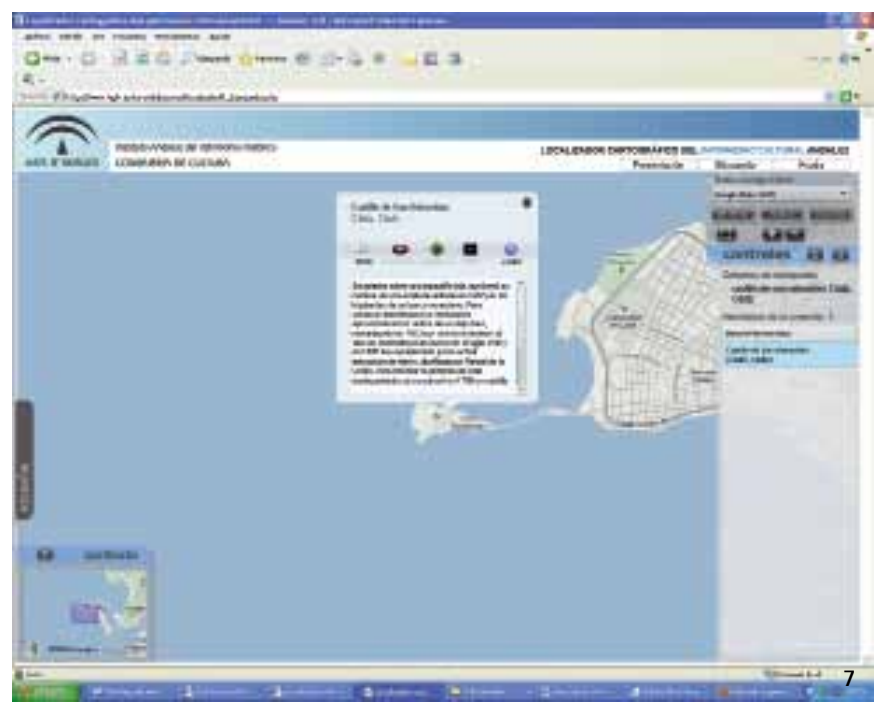

7. Localizador cartográfico del patrimonio cultural andaluz en www. juntadeandalucia.es/cultura/iaph/nav/navegacion.jsp?seccion=PRODUCTOS \&entrada $=/$ portal/Productos/localizador_cartografico

moniales subacuáticos. También la cartografia y vuelos históricos son fuente de datos por contener información para conocer la evolución del espacio geográfico y su relación con las sociedades que lo habitaron.

Pero la verdadera innovación de las fuentes está viniendo de la codificación de toda clase de entidades espaciales a la que referir cierta información, pero de forma muy significativa a direcciones postales. Esto equivale a decir que todo tipo de registro administrativo que tenga asociada una dirección postal adquiere el valor de dato geográfico mediante el adecuado tratamiento informático. Las posibilidades que esto representa son enormes, y supone también que la geoinformación sale del ámbito de las políticas territoriales para interesar al conjunto de las políticas públicas que pueden aportar servicios de interés ciudadano, y a su vez servirse de otros datos que están correlacionados para mejorar su propia gestión.

Los repertorios de información espacial abarcan un amplio espectro de la realidad, casi la totalidad podriamos decir cuando, finalmente, toda información asociada a una dirección postal o cualquier otra entidad espacial se conviertan en geoinformación. Las posibilidades que esto ofrece son para determinados ámbitos de investigación o gestión relativamente desconocidas, de ahí que el incremento de geoinformación por esta vía constituya una de las líneas de trabajo de la que se han de esperar mayores avances. InspirE (DIRECTIVA 2007/2/CE) y también su transposición mediante la reciente LEY 14/2010 establecen una relación de temas exhaustiva, aunque presenta un sesgo ambiental a cuya politica responde. De hecho, InspirE no hace referencia explícita al patrimonio cultural, olvido que en parte subsana la Ley al incluir otros datos "específicos" que proporcionan una imagen cartográfica de las características físicas, jurídicas, económicas, etc., y entre ellas las patrimoniales y culturales. No es el caso del Plan Cartográfico, que hace una adaptación más libre y ajustada a las necesidades 
del conjunto del Sistema Cartográfico de Andalucia, y en este sentido hace referencia explícita a los espacios y bienes protegidos incluidos en el Catálogo General del Patrimonio Histórico Andaluz como datos espaciales que deberán tener garantizadas su catalogación, mantenimiento y accesibilidad.

Este amplio espectro temático está a su vez conectado con la evolución de un modelo cartográfico centralizado y restrictivo, a un modelo de responsabilidades distribuidas entre los diferentes departamentos en función de sus competencias, en virtud de las cuales les corresponde, asimismo, el mantenimiento de los datos espaciales de su ámbito de gestión. Éste es el principio del Dato Único Institucional establecido en el Plan, que trae como consecuencia la unicidad y actualidad de la información espacial servida por cada organismo responsable y que debe quedar asociado, para mayor garantía y eficacia, a los respectivos procesos de su gestión.

\section{INFRAESTRUCTURAS Y SISTEMAS DE INFORMACIÓN GEOGRÁFICA}

La importante producción de datos espaciales temáticos por cada vez más usuarios ha requerido su estructuración en Sistemas de Información Geográfica (SIG), entendidos como el conjunto de recursos tecnológicos y humanos capaz de gestionar la información, operando con la componente espacial de cualquier objeto y sus atributos. En una primera etapa, las enormes ventajas que ofrecen estas herramientas supusieron una proliferación de aplicaciones tan amplia y diversa como lo son las necesidades de sus usuarios. Cada cual por su cuenta o en el reducido ámbito de su trabajo específico ha ido adaptándose a este mundo, familiarizándose con las herramientas para explotar sus enormes potencialidades. Con la puesta en común de experiencias y necesidades, se ha venido comprobando las limitaciones de operar desde su particular repositorio de información, dependiente a su vez del software y de los formatos de archivos utilizados. No sólo hay mucha información de uso común, también hay otra con la que, estableciendo las relaciones pertinentes, podemos enriquecer los análisis y comprender mejor cualquier fenómeno. La integración de la información fue una aspiración que iba alcanzando a círculos cada vez más extensos, a organizaciones más amplias $y$, por consiguiente, con requerimientos técnicos de mayor complejidad y capacidad. Progresivamente hemos venido avanzando hacia estructuras más sólidas y con un carácter más transversal. Así, fueron implantándose SIG departamentales o corporativos en numerosos organismos públicos y privados, que a su vez suponían una nueva ampliación de la base de usuarios. De nuevo, la trayectoria de los departamentos responsables de la política del patrimonio cultural es al mismo tiempo significativa y ejemplar.

Consecuentemente, gran parte de los últimos esfuerzos tienen por objetivo el desarrollo de instrumentos que permitan avanzar en la integración de realidades departamentales más o menos consolidadas a partir de fortalecer las relaciones de coordinación y cooperación con dos objetivos: el desarrollo de herramientas corporativas, en software libre, para facilitar los trabajos, y la puesta en común de los datos espaciales generados por los diferentes departamentos mediante su inclusión en el Catálogo de Datos Espaciales y su difusión a través de servicios interoperables en el marco de las Infraestructuras de Datos Espaciales (IDE).

Precisamente son las plataformas IDE, también IDEAndalucia, las que están adquiriendo el máximo protagonismo en el acceso a la información, aunque todavía sigue siendo importante la publicación de productos tanto en soporte analógico como digital. Las IDE son la respuesta institucional a la creciente demanda de acceso a la información y tiene su fundamento normativo en diversas directivas europeas que, aunque en su origen están vinculadas a la política ambiental, podemos considerarlas que forman parte de una cultura administrativa en la que la transparencia y accesibilidad son consustanciales al servicio público. Para que los datos espaciales sean accesibles a través de los nodos IDE es una condición su previa catalogación mediante la descripción de su contenido y características de calidad, formato, etc. (metadatos), que puedan facilitar a usuarios y aplicaciones localizarlos. En un sistema distribuido como es el de las IDE, con condiciones de interoperabilidad, productos y servicios requieren modelos de datos, metadatos, normas técnicas y estándares. Éste es el momento en el que estamos; no parece reversible y los plazos establecidos no muy extensos. En la actualidad estamos en proceso de catalogación de la información producida conforme a dichos requisitos y elaborando sus metadatos, y prueba de esta actividad, a la se está dedicando importantes recursos, es que están en la propia IDEAndalucia, a la que se van incorporado nodos de diferentes departamentos ${ }^{12}$.

Como consecuencia de esta politica de accesibilidad ya se está alumbrando nuevos horizontes por quienes no se conforman con acceder a la información de forma pasiva, como mero observador que sólo puede ver, consultar y, en el mejor de los casos, descargarse cierta información. Reclama además poder crear, editar y cargar datos espaciales en la Web, elaborar cartografía y compartirla aplicando los estándares establecidos. En definitiva, que los flujos de información sean en doble dirección, concepto básico de lo que se conoce como IDE 2.0. De esta accesibilidad a la información hay que esperar, de hecho ya es así, un incremento de información y con más valor añadido derivado de su utilización por agentes de muy diversa procedencia. El axioma se está cumpliendo: a mayor uso, mayor valor añadido, y, por tanto, más conocimiento y riqueza social. Los cambios no sólo proceden de la esfera tecnológica, sino que está anclada en las propias demandas y usos sociales. Hasta tal punto, que la verdadera brecha digital proviene no tanto de disponer recursos e información, sino de generar servicios para distribuirla en condiciones que permitan su utilización.

Aunque se han hecho algunas referencias a la politica del patrimonio cultural, será en las colaboraciones de este $\mathrm{PH}$ monográfico donde se podrá verificar el alto nivel de aplicación de las tecnologías de la información geográfica a este ámbito. 
${ }^{1}$ Es interesante la etimología de esta palabra compuesta que procede del latín (perceptio): de capere (capturar) y la terminación tio (-ción) que le aporta el significado de acción, efecto, y el prefijo per que denota intensidad. Quiere decir que manejamos mucha menos información que estímulos sensoriales recibimos. Percibir es la función humana que selecciona esa información multisensorial que la inteligencia transfigura: "No se trata de que veamos las cosas y luego las interpretemos, sino que la inteligencia parece funcionar al revés: vemos desde e significado" (MARINA, 2000: 31).

2 Zumthor (1994), a lo largo de su libro, ofrece numerosos ejemplos de la semántica de las palabras en relación con el espacio y todas las nociones a él asociadas.

${ }^{3}$ Harley (2005: 219-228) ha estudiado el papel de la cartografía en la colonización del norte de América, y entre sus prácticas, por ejemplo, la manipulación de la toponimia autóctona, o la identificación únicamente de los asentamientos coloniales dándose a entender que el resto del territorio es virgen. Esta misma idea esta en ese estilo breve y elocuente con que Eduardo Galeano relata sus pensamientos: "Cuenta la historia oficial que Vasco Núñez de Balboa fue el primer hombre que vio, desde una cumbre de Panamá, los dos océanos. Los que alli vivían ¿eran ciegos?" (GALEANO, 2008: 120).

${ }^{4}$ La cita de Almeida (1998: 30) es elocuente: "En cuanto signo representante, o Representamen, un mapa es un diagrama, fatalmente bidimensional. Como diagrama, sólo puede representar contornos. Por tener sólo dos dimensiones, necesita una tercera dimensión (un Interpretante) para ser considerado. Esa es la teoría de Peirce. Ahora bien, el estilo intelectual de Borges tiende a una sistemática reductio ad absurdum de las teorias que adopta. En este caso, como diagrama, el mapa puede ser llevado a un progresivo relleno de sus puntos, hasta convertirse en continuum, dejando asi de ser diagrama para convertirse en 'imagen.' Por otra parte, como figura bidimensional, un mapa puede ser ampliado hasta cubrir totalmente el territorio que lo contiene, aboliendo así la posibilidad de un Interpretante y redefiniendo al mundo como bidimensional".

${ }^{5}$ Estas reflexiones de Fray Mauro vienen al caso: "Para completar un mapa exacto del mundo debo aprender a enfocar el problema desde otra perspectiva. En vez de intentar definir cada continente de un modo que fije su realidad para que todo concuerde con mi interpretación, necesito ser más circunspecto en mis afirmaciones. Cada uno de nosotros tiene derecho a hablar de su litoral, sus montañas, sus desiertos, ninguno de los cuales se ajusta a los de otros. Estamos obligados individualmente a hacer un mapa de nuestro propio pais natal, nuestro propio campo o prado. Llevamos grabado en nuestros corazones el mapa del mundo ta como lo conocemos. Luego empezamos a cubrir el mundo con impresiones de lo que hemos vivido... El mapa que trazamos se convierte en una representación de esas impresiones, contribuyendo cada uno a esa imagen sublime que creemos que existe pero que hasta ahora aún no se ha descubierto" (COWAN, 1997: 141-142).

${ }^{6}$ Un análisis semiológico de algunos de los mapas elaborados con tal diversidad de concepciones espaciales y gráficos (una leyenda sólo de letras, un cosmos local, textos siguiendo las formas topográficas, los centros del mundo...) lo hace Vayssière (1980: 167-177).

7 El mismo vocablo "geografía" (del griego geo=tierra, grafein=dibujo) revela el carácter originalmente indisociable de la Cartografía y la Geografía. La suerte que corrió esta disciplina, disociada en la práctica de las técnicas cartográficas, está siendo en los últimos tiempos felizmente reestablecidas.

${ }^{8}$ Título de la última obra de recopilación de Harley (2005), de la que existe una interesante reseña realizada por Capdevila (2002) a su edición original.

${ }^{9}$ Existe una extraordinaria producción científica en este campo de investigación, pero será suficiente citar a Lois (2009) y García Rojas (2008), donde también se puede encontrar abundantes referencias bibliográficas y sitios Web.

${ }^{10}$ La consideración es oportuna porque en cierta historiografía bien asentada existen abundantes ejemplos de esta mistificación del territorio. No es el caso de la reciente obra Atlas de la Historia del Territorio de Andalucía, que previene acertadamente de este riesgo (DÍAZ QUIDIELLO, 2009: 15-17).

${ }^{11}$ Una recopilación de la normativa sobre esta materia en el ámbito europeo, estatal y autonómico puede consultarse en la Web IDEE: <http://www.idee.es/show. do?to=marco_legal.ES> [consulta: 12/11/2010]

${ }^{12}$ El Geoportal IDEAndalucia ofrece en la actualidad 172 servicios de visualización y 20 de descargas, y recibe una media de 35.000 visitas mensuales.
ALMEIDA, I. (1998) Conjeturas y Mapas: Kant, Peirce, Borges y las geografías del pensamiento. Variaciones Borges: Revista del Centro de Estudios y documentación Jorge Luis Borges [en línea], n. 5 5, 1998, 7-36 <http://www.comunidadrussell.com/ contenidos/textos/download/conjeturasymapas.pdf> [consulta: 05/11/2010]

CAPDEVILA I SUBIRANA, J. (2002) Harley, J. B. The new nature of maps: essays in the history of cartography. Biblio 3W, Revista Bibliográfica de Geografía y Ciencias Sociales [en línea], Universidad de Barcelona. v. VII, n. ${ }^{\circ}$ 404, 15 de octubre de 2002 <http://www.ub.es/geocrit/b3w-404.htm> [consulta: 20/10/2010]

COWAN, J. (1997) El sueño de un cartógrafo. Las meditaciones de fray Mauro, cartógrafo de la corte de Venecia. Barcelona: Ediciones Península, 1997 DÍAZ QUIDIELLO, J. (coord.) (2009) Atlas de la historia del territorio de Andalucia. Sevilla: Consejeria de Vivienda y Ordenación del Territorio, 2009

DIEG0, E. DE (2008) Contra el mapa (Disturbios en la geografía colonial de Occidente). Madrid: Ediciones Siruela, 2008

DIRECTIVA 2007/2/CE del Parlamento Europeo y del Consejo de 14 de marzo de 2007 por la que se establece una infraestructura de información espacial en la Comunidad Europea (Inspire). DOUE, 25 de abril de 2007

FREITAG, U. (1980) Peuples sans cartes. En Cartes et figures de la terre. Paris: Centre Georges Pompidou, 1980, pp. 61-63

GALEANO, E. (2008) Espejos. Una historia casi universal. Madrid: Editorial Siglo $X X I, 2008$

GARCÍA ROJAS, I. B. (2008) La historia de la cartografía. Takwá, revista de Historia [en linea], n. ${ }^{13}$, 2008, pp. 11-32 <http://www.publicaciones.cucsh.udg.mx/pperiod/ takwa/volumenes/tak13.htm> [consulta: 14/11/2010]

HARLEY, J. B. (2005) La nueva naturaleza de los mapas. Ensayos sobre la historia de la cartografía. México: Fondo de Cultura Económica, 2005

LEY 14/2010, de 5 de julio, sobre las infraestructuras y los servicios de información geográfica en España. Boletín Oficial del Estado, 6 de julio de 2010, n.o 163 LOIS, C. (2009) Imagen cartográfica e imaginarios geográficos. Los lugares y las formas de los mapas en nuestra cultura visual. Scripta Nova. Revista Electrónica de Geografia y Ciencias Sociales [en línea], 1 de septiembre de 2009, v. XIII, n. 298 <http://www.ub.es/geocrit/sn/sn-298.htm> [consulta: 14/11/2010] LÓPEZ-GALIACHO, E. (2010) Mapas de las nuevas geografias. Fronterad revista digital [en línea] <http://www.fronterad.com/?q=mapas-galiacho\&page=0,0> [consulta: 14/11/2010]

MARINA, J. A. (2000) Teoría de la inteligencia creadora. Barcelona: Anagrama, 2000

MUMFORD, L. (1977) Técnica y Civilización. Madrid: Alianza, 4ª edición, 1977 OJEDA ZÚJAR J. (2000) La representación cartográfica del territorio de la Comunidad Autónoma de Andalucía. En Atlas de Andalucía. Sevilla: Consejería de Obras Públicas y Vivienda, 2000, pp. 23-57

PLAN Cartográfico de Andalucía 2009-2012 (2008) [en línea]. Junta de Andalucia. Sevilla: Consejeria de Vivienda y Ordenación del Territorio, $2008<$ http://www. juntadeandalucia.es/viviendayordenaciondelterritorio/www/estaticas/cartografia/ plan_cartografico/Plan.pdf> [consulta: 14/11/2010]

THROWER, N. J. W. (2002) Mapas y civilización. Historia de la cartografía en su contexto cultural y social. Barcelona: Ediciones del Serbal, 2002

VAYSSIÈRE, B. H. (1980) Des cartes en Espagne. En Cartes et figures de la terre. Paris: Centre Georges Pompidou, 1980, pp. 167-177

ZUMTHOR, P. (1994) La medida del mundo. Representación del espacio en la Edad Media. Madrid: Cátedra, 1994 